\title{
A risk-based approach to validation of ion chromatography methods using suppressed conductivity
}

Deanna J. Nelson ${ }^{1 *}$ (i) and G. Dean Marbury ${ }^{2}$

\begin{abstract}
Ion chromatography (IC) has evolved into one of the most widely used separation techniques of analytical chemistry. Consequently, the number of users of this method is continuously growing. Analysts often assume that widely used guidelines for HPLC method validation in regulated environments routinely apply to IC. This manuscript provides an analysis of the potential shortcomings of traditional approaches to development and validation of IC methods using suppressed conductivity detection and a risk-based alternative approach to these activities. The goal of the alternative approach is a reduction in the risk of erroneous determinations of analytes when IC methods using suppressed conductivity detection are employed.
\end{abstract}

Keywords: Ion chromatography, Suppressed conductivity, Non-linearity, Risk analysis

\section{Introduction}

Since its introduction in 1975, ion chromatography (IC) has matured into an important analytical methodology having diverse applications in pharmaceutical analysis, medicinal chemistry, environmental chemistry, and materials science (Weiss, 2016). IC complements the more commonly used reversed-phase and normal-phase high performance liquid chromatography (HPLC) and spectroscopic approaches for the separation, identification, and quantitation of analytes. For example, IC has been used for determining inorganic anions and cations, organic acids, carbohydrates, sugar alcohols, proteins, and aminoglycosides (Weiss, 2016).

IC involves separations performed with a column containing an ion exchange stationary phase, and ion detection using a variety of electrochemical and spectroscopic methods (Weiss, 2016). As a result of this diversity, IC offers the ability to determine ionic analytes that have little or no inherent UV absorbance in the presence

*Correspondence: dnelson@phosveda.com

${ }^{1}$ BioLink Life Sciences, Inc., Cary, NC, USA

Full list of author information is available at the end of the article of non-ionic components. In general, the concentration ranges of analyte(s) that may be monitored by IC match the ranges used in conventional HPLC. Therefore, applications of IC include the characterization of active ingredients, excipients and other product components, degradation products and/or impurities, and process and/or waste stream components.

Because IC is a liquid chromatographic method, analysts in the pharmaceutical sciences often assume that widely used ICH, FDA, and USP guidelines for validation of chromatographic assay methods routinely apply (European Medicines Agency, 1995; U.S. Department of Health and Human Services, Food and Drug Administration (FDA), Center for Drug Evaluation and Research (CDER), 2015; U.S. Pharmacopeia, 2007). Specifically, the common validation parameters (e.g., linearity, accuracy, precision, specificity, sensitivity, and robustness) are routinely monitored and validated for IC applications in the pharmaceutical industry and other regulated environments.

This approach often works as expected when the detection method is spectroscopic. However, when suppressed conductivity is employed as the means of detection and 
quantitation, traditional validation approaches may fail. Specifically, despite the observation that linear regression of the IC results often gives very high correlation coefficients (i.e., $r>0.99$ ), the signal (e.g., peak height or area) is not a linear function of the injected mass of the analyte over the broad ranges in analyte concentration that are typically evaluated using HPLC. As a result, the error associated with the method may exceed typical required limits for percent recovery across the concentration range to an extent that accuracy cannot be demonstrated.

The non-linear response between ion concentration and conductivity, a major drawback of IC using suppressed conductivity detection, has been extensively investigated. The goal of these investigations is identification of methodology that enables minimization of error.

The non-linearity problem was first reported early in the 1980s (Slanina et al., 1981; Van Os et al., 1982; Van Os et al., 1984). Van Os et al., for example, reported that attempts to use wide calibration ranges for inorganic analytes in rainwater failed to provide accurate results (Van Os et al., 1984). These investigators noted that the problem arises when a conductometric cell is used as a universal detector in combination with a suppressor. They postulated that the eluate from the suppressor column, a weak acid, makes a contribution to conductivity that decreases as sample concentration is increased. In a subsequent study, the resulting deviations from linearity were addressed by elaborate studies that measured total conductivity and precisely known physical constants: equivalent conductivities of all the ions, dissociation constants of the acids, the cell constant, and the resin capacity factor. Data were "linearized" by calculating the instantaneous analyte concentration in the eluate at a series of points on the chromatographic peak and then integrating to give the mass injected. DouryBerthod et al. showed that the deviation from linearity principally depended on the acid dissociation constant (Ka) of the eluent acid formed in the suppressor (DouryBerthod et al., 1985). Shortly thereafter, Midgley and Parker provided practical approaches for error minimization (Midgley \& Parker, 1989). Their recommended approaches included (a) direct reading of concentrations off a calibration curve; (b) treating calibration as a series of linear segments (i.e., a "point to point" calibration); (c) fitting calibration to second- or higher order equations; (d) changing the injection volume to accommodate deviations from linearity; and (e) changing the eluent.

All of these early disclosures related to anion analysis using a weak base as an eluent (e.g., carbonate), because the extent of non-linearity appeared to be proportional to the Ka of the suppressed eluent (Lucy, 1998; Achilli \& Romele, 1997; Tartari et al., 1995; Costa Pessoa et al., 1992; Brinkmann et al., 2002). Therefore, it was suggested that linearity would be significantly improved if a strong base was substituted as the eluent. However, Brinkmann et al. showed that non-linear analyte responses persisted even with use of sodium hydroxide eluents and recommended the use of quadratic functions for calibration (Brinkmann et al., 2002). However, the Brinkmann team also acknowledged that for routine laboratory work, it is not feasible to model complicated calibration curves similar to those which are routinely observed, nor is it possible to control all of the parameters that are involved in IC analysis.

Recently Eom et al. echoed Brinkmann's conclusions by acknowledging that the electrical conductivity response of an anion in suppressed IC with a hydroxide eluent is highly variable. Keeping the carbonate level below 0.1 $\mu \mathrm{mol} / \mathrm{L}$ is of prime importance but may not be sufficient to remove non-linearity. As an alternative, they proposed adding a low concentration of a strong acid suppressant such as $0.5 \mu \mathrm{mol} / \mathrm{L} p$-toluenesulfonate, a deliberate additive that was reported to improve calibration linearity in IC using an isocratic elution mode (Eom et al., 2015).

In contrast to IC with basic eluents, some authors have assumed that when a strong acid was used as the eluent, calibrations for cation analysis were essentially linear. Eom et al. showed that this assumption is incorrect (Eom \& Lee, 2016). In order to minimize errors introduced by non-linear responses, they recommended either using two or more linear segments (i.e., a "point-to-point" calibration model) or a second-order curve fitting.

Weiss has analyzed the conditions under which ion conductivity is directly related to ion concentration and compared them to conditions under which increasing electrolyte concentration introduce error (Weiss, 2016). Weiss postulates that conductivity is a useful measure of concentration when:

- The electrolyte is fully dissociated.

- Only electrostatic forces acting between ions are responsible for interionic interactions.

- The electrostatic interaction energy is small compared to the thermal energy.

- The ions are regarded as point charges with an electric field of spherical symmetry and thus are nonpolarizable.

- The dielectric constant of the electrolyte solution is equal to that of the pure solvent.

These conditions apply when the concentration of the analyte is low and nears infinite dilution. However, as the analyte concentration increases, the following interactions come into play: 
- Ion pairs may form, reducing the effective concentration.

- Ion-molecule interactions may occur and alter the solvation state of the ions and the solvent structure.

- Thermal motion is no longer unhindered.

- Interactions between ions may cause ion polarization, altering the electric field.

- Interactions of ions with the solvent change the dielectric constant of the solvent.

Thus, the analyst faces the quandary of reducing the analyte concentration sufficiently to minimize interactions that introduce error but maintaining an analyte concentration sufficiently high to enable identification and quantitation of the ion in the presence of potential interferents. In addition, the analyst must assess the impact that use of atypical models will have on the ability to transfer and employ the assay in multiple laboratories.

Many regulatory bodies (including the U.S. FDA) accept regression models that are non-linear. However, we anticipated that the analytical method for succinate assay that we developed and validated would be used in multiple laboratories by analysts having a range of skill levels. For this reason, we were reluctant to proceed with either a "point-to-point" segmental calibration model or a non-linear regression model, since either might subsequently prove difficult to transfer and use in laboratories other than our own.

\section{A new perspective on method development}

With the foregoing discussion in mind, we considered an alternate, risk-based approach to method development that embodies the principals set forth in ISO/IEC 17025:2017 (Technical Committee ISO/CASCO, 2017). This guidance recommends the application of statistical techniques to the planning of procedures for ensuring the validity of results. Specifically, in this case, plans for IC method development and validation using suppressed conductivity detection were structured to reduce the risk of analytical error due to method non-linearity while addressing three questions:

1. What is the target value that must be valid in order for an assay to meet analyte specifications?

2. What is the concentration range around the target value that must be valid in order for an assay to meet analyte specifications?

3. What is the concentration range around the target value that will clearly and unequivocally verify that a determination is valid but falls outside of a concentration range cited in the analyte specifications?
Once the target working concentration and related concentration ranges are determined, method linearity is assessed and the calibration curve is redefined to ensure linearity and accuracy over a narrower range of analyte concentrations.

An illustration of an application of this approach is presented below.

\section{Example of a risk-based approach to IC method development and validation}

An assay for succinate was required as part of commercial development of calcium succinate monohydrate and its encapsulated formulations.

\section{Materials and methods}

An IC method was developed using a ThermoFisher Scientific Dionex ${ }^{\mathrm{TM}}$ Aquion $^{\mathrm{TM}}$ IC system using suppressed conductivity detection. The HPLC system was set up as follows:

Flow rate: $1.0 \mathrm{~mL} / \mathrm{min}$

Injection volume: $10 \mu \mathrm{L}$

Column temperature: $30{ }^{\circ} \mathrm{C}$

Detection: Conductivity mode

Detector settings: Cell temp: $35^{\circ} \mathrm{C}$

AERS 500:50 mA

Guard column: Dionex IonPac AG11-HC, $4 \times 50 \mathrm{~mm}$

Column: Dionex IonPac AS11-HC, $4 \times 250 \mathrm{~mm}$

Acquisition time: Approximately $9 \mathrm{~min}$

The mobile phase, $20 \mathrm{mM}$ sodium hydroxide, was delivered isocratically at a flow rate of $1.0 \mathrm{~mL} / \mathrm{min}$. Both solvent- and online-degassing were used to minimize interference by the carbonate peak at $5.6 \mathrm{~min}$, which was resolved from the succinate peak at $5.2 \mathrm{~min}$.

\section{Results}

A typical chromatogram is shown in Fig. 1.

During assay development, a succinate concentration of $50 \mu \mathrm{g} / \mathrm{mL}$ was identified as a concentration sufficiently large to give a characteristic response that was significantly higher than the limits of quantitation and detection. In addition, this succinate concentration was significantly lower than the analyte's maximum concentration in water/eluent and an analyte concentration that would saturate suppression. As part of development, linearity, precision, accuracy, specificity, and robustness were assessed and appeared to be acceptable sufficient to proceed with validation.

Initially, linearity was evaluated over the range of 20 $\mu \mathrm{g}$ succinate $/ \mathrm{mL}$ to $200 \mu \mathrm{g}$ succinate $/ \mathrm{mL}$. A calibration curve was plotted (Fig. 2) using the peak areas and each corresponding concentration and the correlation coefficient was calculated. 


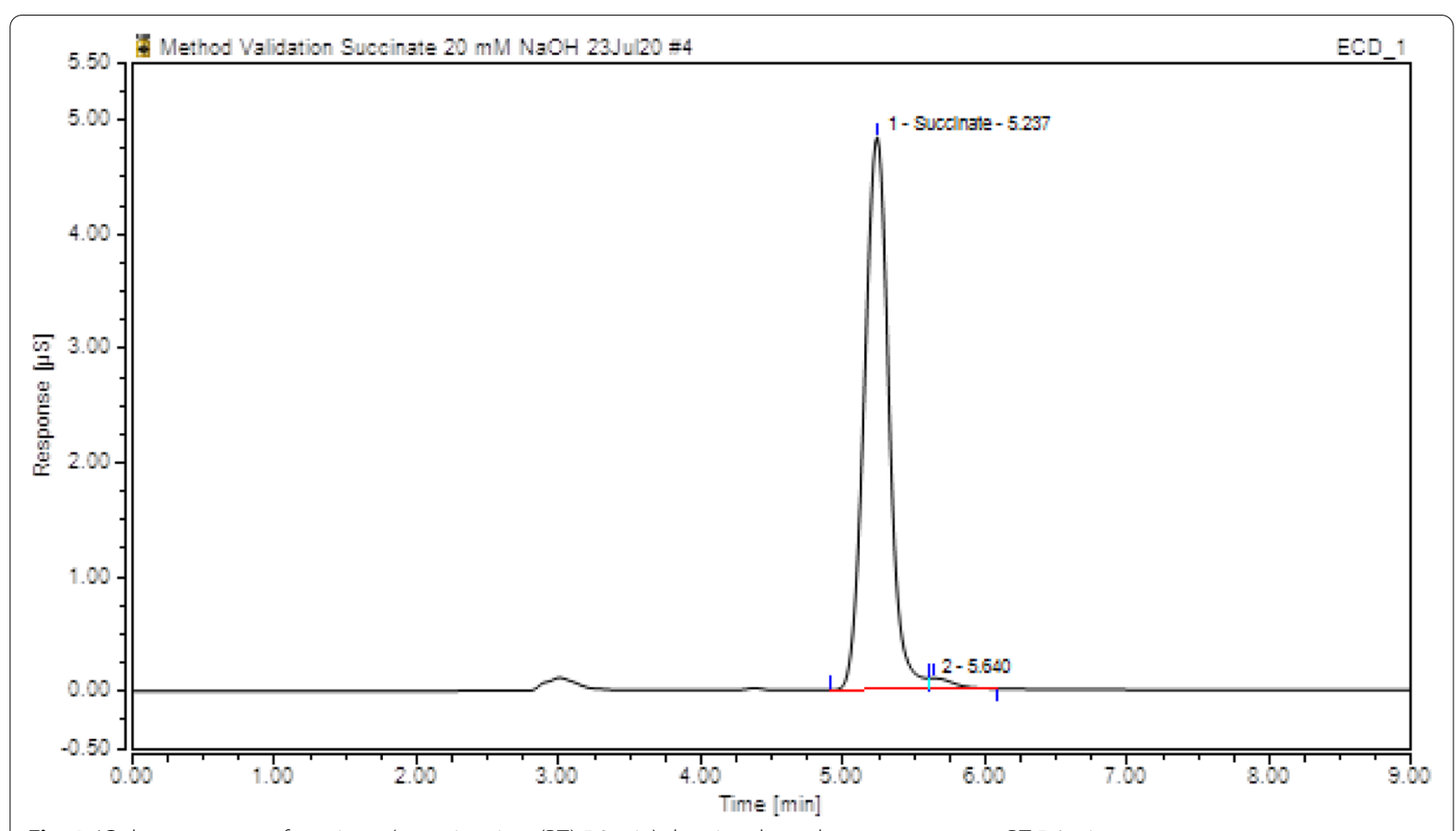

Fig. 1 IC chromatogram of succinate (retention time (RT) $5.2 \mathrm{~min}$ ) showing the carbonate response at RT $5.6 \mathrm{~min}$

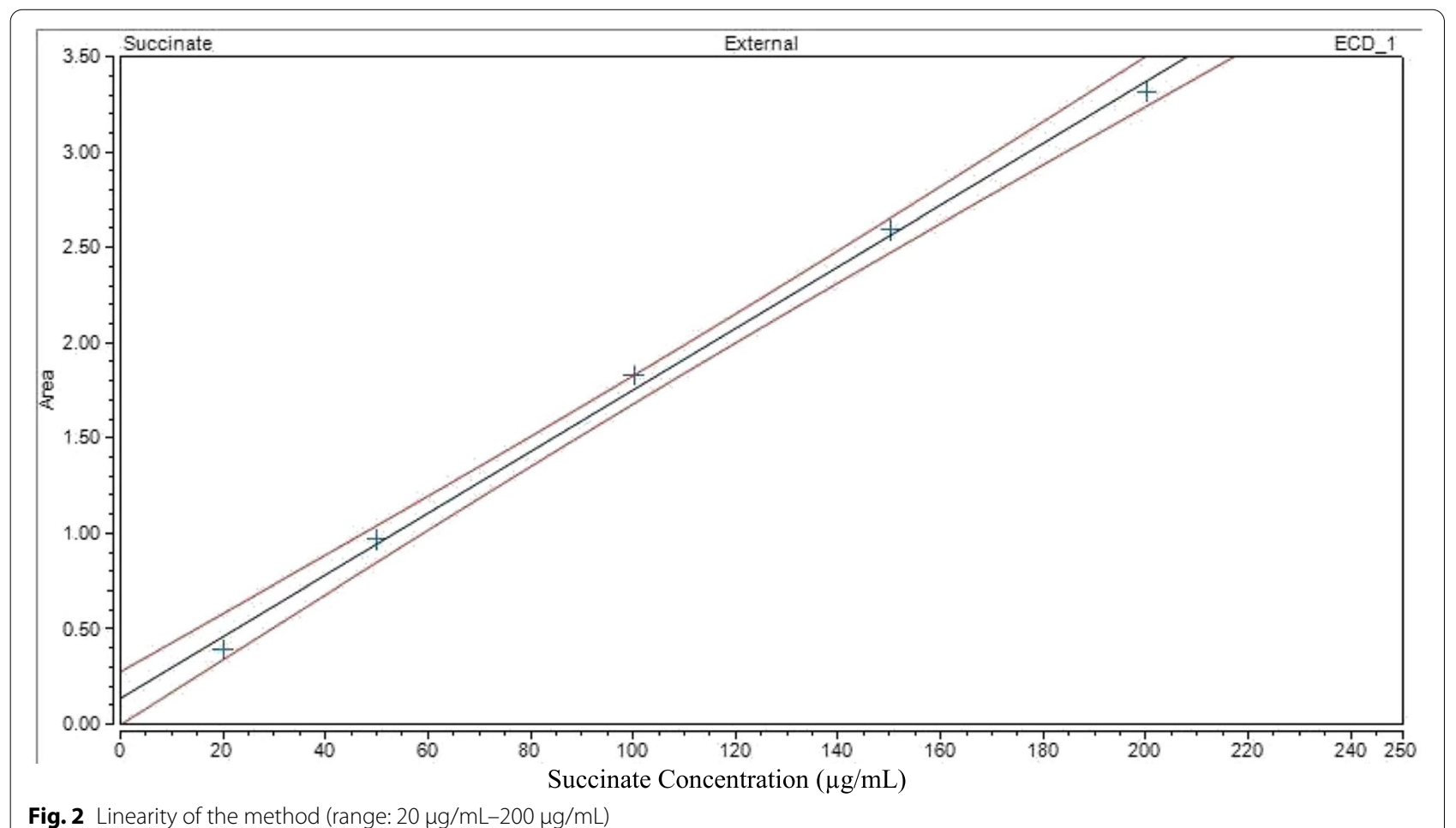

Fig. 2 Linearity of the method (range: $20 \mu \mathrm{g} / \mathrm{mL}-200 \mu \mathrm{g} / \mathrm{mL}$ ) 
Table 1 provides the regression parameters. The acceptance criteria were twofold: (a) the method is linear over the expected concentration range; and (b) Pearson's correlation coefficient $(r)$ is $\geq 0.995$. The data in Table 1 indicate that the method is linear over the range $20 \mu \mathrm{g} / \mathrm{mL}$ to $200 \mu \mathrm{g} / \mathrm{mL}$. Pearson's correlation coefficient $(r)$ is 0.9986 . Thus, all results pass the acceptance criteria.

Method precision was evaluated by performing the method using six independent preparations of succinate at the $50 \mu \mathrm{g} / \mathrm{mL}$ succinate concentration. The concentration found was calculated using the peak areas of the six preparations and the calibration curve regression parameters given in Table 2.

The acceptance criterion for method precision required that the RSD of the peak area was $\leq 10 \%$. The data in Table 2 confirm that this criterion was satisfied.

Assessment of method accuracy during method development, however, failed to meet acceptance criteria. As is typical, the accuracy was evaluated at $50 \%$, $100 \%$, and $150 \%$ of the nominal concentration of $50 \mu \mathrm{g} /$ $\mathrm{mL}$. Two acceptance criteria applied: (1) the recovery at each level is $90-110 \%$; and (2) the RSD at each level is $\leq 10 \%$. When the sample concentration was calculated using the response factors determined from the regression parameters (Table 3), it became clear that an assumption of linearity over this broad concentration range might introduce errors sufficiently large to cause inappropriate acceptance or rejection of calcium succinate drug substance or drug product.

A response factor was calculated at each concentration as the ratio of the peak area to the concentration of the standard at that concentration. Examination of the response factor at each concentration level in Table 1 revealed the subtle non-linearity of the method across this concentration range, especially in the 50-200 $\mu \mathrm{g} /$ $\mathrm{mL}$ range (Fig. 3). The differences in response factor are directly related to the inaccuracies exhibited by the analysis.

Table 1 Succinate linearity (range $20 \mu \mathrm{g} / \mathrm{mL}$ to $200 \mu \mathrm{g} / \mathrm{mL}$ )

\begin{tabular}{lll}
\hline Calibration level & Concentration $(\boldsymbol{\mu g} / \mathbf{m L})$ & Area \\
\hline 1 & 20.03 & 0.391 \\
2 & 50.07 & 0.968 \\
3 & 100.14 & 1.830 \\
4 & 150.21 & 2.594 \\
5 & 200.28 & 3.317 \\
Slope & 0.0162 & \\
Intercept & 0.1358 & \\
$r$ & 0.9986 & \\
\hline
\end{tabular}

Table 2 Method precision results

\begin{tabular}{lllll}
\hline Rep & $\begin{array}{l}\text { Theoretical } \\
\text { concentration }(\boldsymbol{\mu g} / \\
\mathbf{m L})\end{array}$ & Area & $\begin{array}{l}\text { Concentration } \\
\text { found }(\boldsymbol{\mu} \mathbf{g} / \mathbf{m L})\end{array}$ & \% Recovery \\
\hline 1 & 50.03 & 0.972 & 51.68 & 103.4 \\
2 & 50.11 & 0.961 & 50.99 & 101.9 \\
3 & 50.18 & 0.930 & 49.13 & 97.9 \\
4 & 50.01 & 0.951 & 50.39 & 100.8 \\
5 & 50.12 & 0.944 & 49.95 & 99.7 \\
6 & 50.15 & 0.958 & 50.85 & 101.4 \\
Avg & $\mathbf{5 0 . 1}$ & $\mathbf{0 . 9 5 3}$ & $\mathbf{5 0 . 5 0}$ & $\mathbf{1 0 0 . 8}$ \\
SD & $\mathbf{0 . 0 6 7}$ & $\mathbf{0 . 0 1 5}$ & $\mathbf{0 . 8 9 0}$ & $\mathbf{0 . 9 0 2}$ \\
$\%$ RSD & $\mathbf{0 . 1}$ & $\mathbf{1 . 5}$ & $\mathbf{1 . 8}$ & $\mathbf{1 . 8}$ \\
\hline
\end{tabular}

Although the data could be analyzed using second order or higher power equations, we hesitated to employ these non-linear equations because we anticipated that the assay would be transferred to several other laboratories where the validity of this type of correlation might be questioned.

To address this concern and plan for a risk-based approach to setting a calibration range that would exhibit both linearity and accuracy across the range, we answered each of the questions listed above. (Our responses are italicized in the text boxes below.) Note that this approach applies both to concentration ranges for assay and to determination of point-to-point ranges in concentration that might be required for dissolution or bioavailability assays.

1. What is the target concentration that must be valid in order for an assay to meet analyte specifications?

The target succinate concentration was $50 \mathrm{\mu g} / \mathrm{mL}$, a value that represented a succinate concentration that was easily detected and quantitated but different from solution saturation. The concentration was sufficiently high that interference by carbonate, a nearby peak response, was minimal. When dilution factors were considered, the concentration of succinate could be calculated.

Table 3 Risk of error in accuracy results (limit 10\% difference) calculated using linearity regression parameters (range $20 \mu \mathrm{g} / \mathrm{mL}$ to $200 \mu \mathrm{g} / \mathrm{mL}$ )

\begin{tabular}{llllll}
\hline Accuracy level & \multicolumn{6}{l}{$\begin{array}{l}\text { Percent difference in calculated concentration } \\
\text { ( } \boldsymbol{\mu g} / \mathbf{m L}) \text { using response factor at accuracy } \\
\text { level shown }\end{array}$} \\
\cline { 2 - 6 } & $\mathbf{2 0}$ & $\mathbf{5 0}$ & $\mathbf{1 0 0}$ & $\mathbf{1 5 0}$ & $\mathbf{2 0 0}$ \\
\hline $50 \%(25 \mu \mathrm{g} / \mathrm{mL})$ & 3.7 & 2.8 & -2.8 & -4.8 & -13.5 \\
$100 \%(50 \mu \mathrm{g} / \mathrm{mL})$ & 2.6 & 1.6 & -4.1 & -10.1 & -14.8 \\
$150 \%(75 \mu \mathrm{g} / \mathrm{mL})$ & 12.2 & 11.3 & 6.2 & 0.7 & -3.5 \\
\hline
\end{tabular}




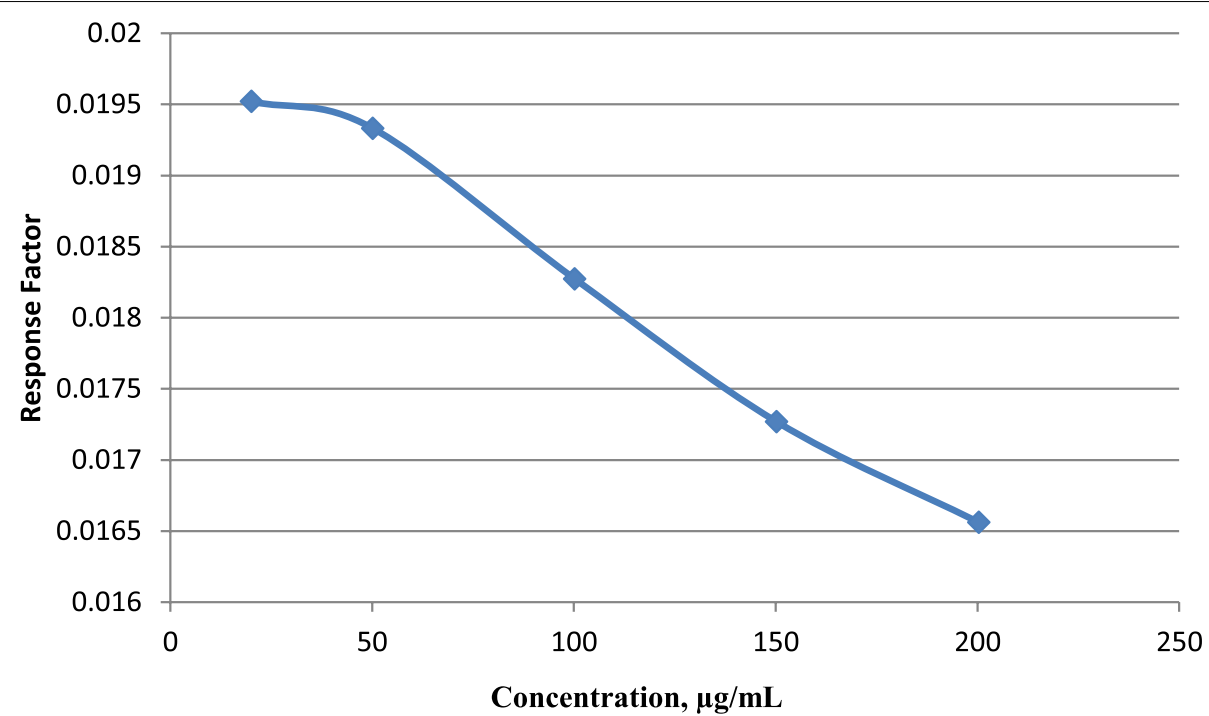

Fig. 3 Response factors as a function of standard concentration (classic approach)

2. What is the concentration range around the target that must be valid in order for an assay to meet specifications associated with the succinate assay?

The limit for succinate determination was set in the material specification as $\pm 10 \%$ of the target value (i.e., 90.0 to $110.0 \%$ of the succinate concentration in calcium succinate monohydrate).

3. What is the concentration range around the target that will clearly and unequivocally verify that a determination is valid but falls outside of a specified succinate concentration range?

In order to set the limits which would encompass both the specification range and a larger range in which the determination would be both accurate and precise, we set limits in the differences in the response factor relative to the response factor at the target concentration $(50 \mathrm{\mu g} / \mathrm{mL})$. The limits we selected were based on the criterion of $\pm 5 \%$ difference in response factor over the concentration range of interest. We used the response factor curve (Fig. 3) to identify the approximate succinate concentrations that would likely meet this criterion. Thus, a succinate concentration range from $35 \mu \mathrm{g} / \mathrm{mL}$ to $65 \mu \mathrm{g} / \mathrm{mL}$ was used.

The risk-based approach was used to calculate the new and narrower succinate concentration range of $35 \mu \mathrm{g} / \mathrm{mL}$ to $65 \mu \mathrm{g} / \mathrm{mL}$ succinate (i.e., $70 \%$ to $130 \%$ of the target working standard concentration of $50 \mu \mathrm{g} /$ $\mathrm{mL})$. Method validation was performed using the typical validation elements. Linearity, precision, and accuracy were determined for succinate concentrations in this range. All other validation elements (i.e., specificity, robustness, and solution stability) used only the 50 $\mu \mathrm{g} / \mathrm{ml}$ succinate working standard, and all associated acceptance criteria were met for those elements.

Linearity was evaluated over the range of $35 \mu \mathrm{g}$ succinate $/ \mathrm{mL}$ to $65 \mu \mathrm{g}$ succinate $/ \mathrm{mL}$ (Table 4 ).

A calibration curve was plotted (Fig. 4) and the regression parameters were calculated.
As expected, the method is linear over the expected concentration range. Pearson's correlation coefficient $(r)$ is $\geq 0.995$.

Method precision was evaluated by performing the method using six independent preparations of succinate at the $50 \mu \mathrm{g} / \mathrm{mL}$ concentration. The percentage relative standard deviation of the peak areas of the six preparations was calculated using the calibration curve regression parameters as given in Table 5 .

The \%RSD of the peak areas for six preparations was $1.5 \%$. All results passed the acceptance criterion

Lastly, results for Accuracy were calculated using the response factor (RF) for the $50 \mu \mathrm{g} / \mathrm{mL}$ working standard as shown in Table 6.

Using this single response factor, the succinate concentrations found were accurate within the validation protocol range of 90 to $110 \%$ potency. For this reason, the method accuracy was considered valid over this entire concentration range.

Moreover, examination of the response factors over this concentration range (Table 4) confirms that all values fell within the range $0.01855 \pm 5 \%$, as anticipated (i.e., we calculated a range from a response factor of 0.01762 to a response factor of 0.01948).

\section{Discussion and conclusions}

The number of analytical methods using ion chromatography continues to grow. As a consequence, more analysts will consider use of IC with suppressed conductivity as the method of choice for determination of an analyte. 
Table 4 Results for linearity and response factor (RF) determinations

\begin{tabular}{|c|c|c|c|c|c|c|}
\hline Concentration $(\mu \mathrm{g} / \mathrm{mL})$ & Area & Statistics & & $\mathrm{RF}$ & Statistic & \\
\hline 35.06 & 0.654 & & & $1.866 \mathrm{E}-02$ & & \\
\hline & 0.652 & & & $1.860 \mathrm{E}-02$ & & \\
\hline & 0.652 & N & 6 & $1.860 \mathrm{E}-02$ & $n$ & 6 \\
\hline & 0.654 & SD & 0.001 & $1.866 \mathrm{E}-02$ & SD & $2.80463 E-05$ \\
\hline & 0.654 & Avg & 0.653 & $1.866 \mathrm{E}-02$ & Avg & $1.86321 \mathrm{E}-02$ \\
\hline & 0.653 & $\%$ RSD & 0.15 & $1.863 \mathrm{E}-02$ & $\%$ RSD & 0.15 \\
\hline 40.06 & 0.735 & & & $1.835 \mathrm{E}-02$ & & \\
\hline & 0.739 & & & $1.845 \mathrm{E}-02$ & & \\
\hline & 0.747 & N & 6 & $1.865 \mathrm{E}-02$ & $n$ & 6 \\
\hline & 0.740 & SD & 0.004 & $1.847 \mathrm{E}-02$ & SD & $9.81621 E-05$ \\
\hline & 0.742 & Avg & 0.741 & $1.852 \mathrm{E}-02$ & Avg & $1.84871 \mathrm{E}-02$ \\
\hline & 0.741 & $\%$ RSD & 0.53 & $1.850 \mathrm{E}-02$ & $\%$ RSD & 0.53 \\
\hline 50.08 & 0.927 & & & $1.851 \mathrm{E}-02$ & & \\
\hline & 0.930 & & & $1.857 \mathrm{E}-02$ & & \\
\hline & 0.928 & $n$ & 6 & $1.853 \mathrm{E}-02$ & $n$ & 6 \\
\hline & 0.930 & SD & 0.001 & 1.857E-02 & SD & 3.09344E-05 \\
\hline & 0.928 & Avg & 0.929 & $1.853 \mathrm{E}-02$ & Avg & $1.85503 \mathrm{E}-02$ \\
\hline & 0.931 & $\%$ RSD & 0.15 & $1.859 \mathrm{E}-02$ & $\%$ RSD & 0.17 \\
\hline 60.10 & 1.092 & & & $1.817 \mathrm{E}-02$ & & \\
\hline & 1.091 & & & $1.815 \mathrm{E}-02$ & & \\
\hline & 1.104 & $n$ & 6 & $1.837 \mathrm{E}-02$ & $n$ & 6 \\
\hline & 1.082 & $S D$ & 0.008 & $1.800 \mathrm{E}-02$ & SD & 0.000133846 \\
\hline & 1.084 & Avg & 1.092 & $1.804 \mathrm{E}-02$ & Avg & $1.81626 \mathrm{E}-02$ \\
\hline & 1.096 & $\%$ RSD & 0.74 & $1.824 \mathrm{E}-02$ & $\%$ RSD & 0.74 \\
\hline 65.10 & 1.172 & & & $1.800 \mathrm{E}-02$ & & \\
\hline & 1.164 & & & $1.788 \mathrm{E}-02$ & & \\
\hline & 1.176 & $n$ & 6 & $1.806 \mathrm{E}-02$ & $\mathrm{n}$ & 6 \\
\hline & 1.171 & SD & 0.004 & $1.799 \mathrm{E}-02$ & SD & $5.96871 \mathrm{E}-05$ \\
\hline & 1.170 & Avg & 1.171 & 1.797E-02 & Avg & $1.79789 \mathrm{E}-02$ \\
\hline & 1.170 & $\%$ RSD & 0.33 & 1.797E-02 & $\%$ RSD & 0.33 \\
\hline
\end{tabular}

Analysts often assume that widely used guidelines for HPLC method validation in regulated environments routinely apply to IC. When the subtle non-linearity in peak response to changes in analyte concentration occurs, the analyst using IC with suppressed conductivity detection will be required to address two key questions: What is the analyte concentration that will provide a peak response sufficiently high to enable reliable identification and quantitation in the presence of potential interferents and yet sufficiently low to minimize ion interactions associated with error? What is the range of concentrations around this target peak response which will provide data that are reliable and reproducible?

Historically, data that were generated with IC and suppressed conductivity detection have been analyzed in three general ways. One group has ignored the subtle non-linearity of the assay and accommodated errors somewhat larger than are accepted for HPLC assays.
A second group has analyzed the data using multiple "point-to-point" segments across the concentration range to reduce error. A third group has employed second- or higher-order equations to correlate peak responses with concentration.

The approach we described in this report provides another alternative for completing assay development in a manner that addresses the non-linearity over broad concentration ranges of analyte responses that are found using IC with suppressed conductivity and reduces the risk of subtle errors in determining analyte concentrations. This approach requires generation and analysis of a peak response-analyte concentration curve over the broad concentration ranges typical of chromatographic assays. Based on these initial data, the analyst selects a target concentration that will be employed as the working standard for the assay or as a target concentration at each point in the extended calibration 


\section{Standard Area Response}

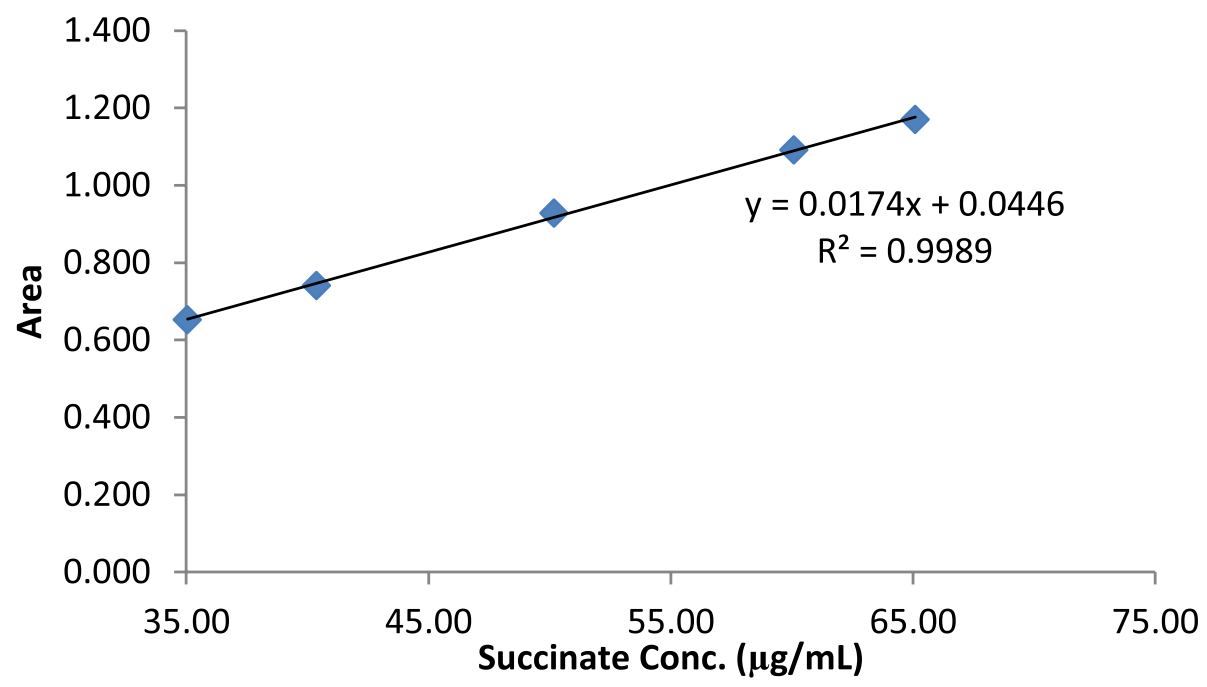

Fig. 4 Area response for working standards ( $35 \mu \mathrm{g} / \mathrm{mL}$ to $65 \mu \mathrm{g} / \mathrm{mL}$ range)

Table 5 Method precision results

\begin{tabular}{lllll}
\hline Rep & $\begin{array}{l}\text { Theoretical } \\
\text { concentration }(\boldsymbol{\mu g} / \\
\mathbf{m L})\end{array}$ & Area & $\begin{array}{l}\text { Concentration } \\
\text { found }(\boldsymbol{\mu g} / \mathbf{m L})\end{array}$ & \% Recovery \\
\hline 1 & 50.03 & 0.927 & 51.68 & 103.4 \\
2 & 50.11 & 0.930 & 50.99 & 101.9 \\
3 & 50.18 & 0.928 & 49.13 & 97.9 \\
4 & 50.01 & 0.930 & 50.39 & 100.8 \\
5 & 50.12 & 0.928 & 49.95 & 99.7 \\
6 & 50.15 & 0.931 & 50.85 & 101.4 \\
Avg & $\mathbf{5 0 . 1}$ & $\mathbf{0 . 9 5 3}$ & $\mathbf{5 0 . 5 0}$ & $\mathbf{1 0 0 . 8}$ \\
SD & $\mathbf{0 . 0 6 7}$ & $\mathbf{0 . 0 1 5}$ & $\mathbf{0 . 8 9 0}$ & $\mathbf{0 . 9 0 2}$ \\
\%RSD & $\mathbf{0 . 1}$ & $\mathbf{1 . 5}$ & $\mathbf{1 . 8}$ & $\mathbf{1 . 8}$ \\
\hline
\end{tabular}

Table 6 Risk of an inaccurate result as a function of concentration calculated using the response factor for the $50 \mathrm{\mu g} /$ $\mathrm{ml}$ working standard (limit 10\% difference)

\begin{tabular}{llllll}
\hline $\begin{array}{l}\text { Actual } \\
\text { conc. }(\boldsymbol{\mu g} / \\
\mathbf{m L})\end{array}$ & Area & RF & $\begin{array}{l}\text { Conc. } \\
\text { found }(\boldsymbol{\mu g} / \\
\mathbf{m L})\end{array}$ & \% Recovery & \% Difference \\
\hline 35.06 & 0.653 & 0.01851 & 35.01 & 100.7 & -0.7 \\
40.37 & 0.741 & 0.01851 & 40.37 & 99.1 & 0.9 \\
50.19 & 0.929 & 0.01851 & 50.19 & 100.0 & 0.0 \\
60.10 & 1.092 & 0.01851 & 60.07 & 98.1 & 1.9 \\
65.10 & 1.171 & 0.01851 & 65.02 & 97.1 & 2.9 \\
\hline
\end{tabular}

curve. Then the acceptance limits associated with the target concentration are defined by examination of the response curve and determination of the variability in response that will be found acceptable for the assay. For our assay, we selected a variability of $\pm 5 \%$ in the response factor. Use of this information enables tentative definition of the limits in analyte concentration associated with the range of response factors. Development is concluded with verification and validation that the data within this narrower range of analyte concentrations reflects accurate and precise analyte determinations. Finally, the assay validation must ensure that the findings enable good decisions about the acceptability of the analyte purity (its assay), or the concentration that is monitored during a dissolution or bioavailability study.

\section{Acknowledgements}

This research did not receive any specific grant from funding agencies in the public, commercial, or not-for-profit sectors.

\section{Authors' contributions}

These authors contributed equally. All authors have given approval to the final version of the manuscript.

\section{Declarations}

Competing interests

The authors declare that there are no conflicts of interest.

Author details

${ }^{1}$ BioLink Life Sciences, Inc., Cary, NC, USA. ${ }^{2}$ Biolink Life Sciences Carolinas, Inc., Cary, NC, USA. 
Received: 16 October 2021 Accepted: 11 November 2021

Published online: 13 December 2021

\section{References}

Achilli M, Romele L (1997) Use of factorial experimental design for the rapid evaluation of main and interactive factors affecting linearity in calibration curves for sulfate analysis by ion chromatography. J Chromatogr A 770:29-37

Brinkmann T, Specht CH, Frimmel FH (2002) Non-linear calibration functions inion chromatography with suppressed conductivity detection using hydroxide eluents. J Chromatogr A 957:99

Costa Pessoa J, Gameiro A, Goncalves MC, Fermino AS (1992) Nonlinearity of calibrations in the analysis of anions by ion chromatography with suppressed conductivity detection. Port Electrochim Acta 10:49-61

Doury-Berthod M, Giampaoli P, Pitsch H, Sella C, Poitrenaud C (1985) Theoretical approach of dual-column ion chromatography. Anal Chem 57:2257-2263

Eom J, Kim MY, Lee B, Lee DS (2015) Lower limits and nonlinearity of calibration in suppressed ion chromatography with strong acid eluent. Bull Kor Chem Soc 35:2073-2080

Eom J, Lee DS (2016) Low-temperature conductivity detection for ultrasensitive ion chromatography. Bull Kor Chem Soc 37:321-329

European Medicines Agency. 1995. ICH Topic Q 2 (R1) - Validation of Analytical Procedures: Text and Methodology, Step 5, Note for Guidance on Validation of Analytical Procedures: Text and Methodology (CPMP/ICH/381/95).

Lucy CA (1998) What are the unanswered (and unasked) questions in ion analysis? J Chromatogr A 804:3-15

Midgley D, Parker RL (1989) Non-linearity of calibration in the determination of anions by ion-chromatography with suppressed conductivity detection. Talanta 36:1277-1283
Slanina J, Bakker FP, Jongejan PAC, Van Lamoen L, Mols JJ (1981) Fast determination of anions by computerized ion chromatography coupled with selective detectors. Anal Chim Acta 130:1-8

Tartari GA, Marchetto A, Mosello R (1995) Precision of ion chromatographic analyses compared with that of other analytical techniques through intercomparison exercises. J Chromatogr A 706:21-29

Technical Committee ISO/CASCO. 2017. ISO/IEC 17025:2017 General requirements for the competence of testing and calibration laboratories.

U.S. Department of Health and Human Services, Food and Drug Administration (FDA), Center for Drug Evaluation and Research (CDER) (2015) Analytical procedures and method validation for drugs and biologics guidance for industry

U.S. Pharmacopeia $(2007)<1225>$ validation of Compendial methods. U.S. Pharmacopeial Convention, Rockville current volume

Van Os MJ, Slanina J, de Ligny CL, Agterdenbos J (1984) Linear calibration in ion chromatography by calculating total amounts of sample from measured conductivity data. Anal Chim Acta 156:169-180

Van Os MJ, Slanina J, de Ligny CL, Hammers WE, Agterdenbos J (1982) Determination of traces of inorganic anions by means of high-performance liquid chromatography on Zipax-sax columns. Anal Chim Acta 144:73-82

Weiss J (2016) Handbook of ion chromatography, 4th edn. Wiley-VCH Verlag GmbH \& Co. KGaA, Weinheim L. Bhattacharyya, J. S. Rohrer (Eds.). Applications of ion chromatography in the analysis of pharmaceutical and biological products. Wiley, Hoboken, NJ, 2012

\section{Publisher's Note}

Springer Nature remains neutral with regard to jurisdictional claims in published maps and institutional affiliations.

\section{Submit your manuscript to a SpringerOpen ${ }^{\circ}$ journal and benefit from:}

- Convenient online submission

- Rigorous peer review

- Open access: articles freely available online

- High visibility within the field

- Retaining the copyright to your article

Submit your next manuscript at springeropen.com 